\title{
Dietary risk factors for hip fracture: an umbrella review of observational evidence
}

Hip fracture is an increasingly prevalent global health issue that impairs quality of life and increases morbidity and mortality in elderly populations ${ }^{(1-3)}$. There is potential for risk reduction through diet modification, but the dietary risk factors for hip fracture have not yet been summarised. This umbrella review aimed to summarise the evidence regarding dietary factors and hip fracture incidence in adults, and to evaluate the methodological quality of systematic reviews and meta-analyses and the quality of evidence for each association.

A systematic literature search was performed in Medline, Embase, Web of Science, and the Cochrane Library up to November 2020. Inclusion criteria were systematic reviews with meta-analyses reporting summary risk estimates for associations between hip fracture incidence and intake of individual foods, food groups, beverages, nutrients, or adherence to dietary patterns. The methodological quality of systematic reviews and meta-analyses was assessed using AMSTAR-2, and the quality of evidence for each association was assessed using GRADE. Results were synthesised descriptively.

Sixteen systematic reviews were identified, covering thirty-four exposures, including dietary patterns ( $\mathrm{n}=2$ meta-analyses), foods, food groups, or beverages $(n=16)$, macronutrients $(n=3)$, and micronutrients $(n=13)$. Identified meta-analyses included 6,282 to 3,730,424 participants with between 322 and 26,168 hip fractures. The methodological quality (AMSTAR-2) of all systematic reviews was low or critically low. The quality of evidence (GRADE) was moderate for an inverse association between hip fracture incidence and intake of fruits and vegetables combined (adjusted relative risk for higher vs lower intakes: 0.92 [95\% confidence interval: 0.87 to $0.98]$ ), and very low for the remaining thirty-three exposures.

Dietary factors may play a role in the primary prevention of hip fracture, but the methodological quality of systematic reviews and meta-analyses was below international standards, and there was a lack of high-quality evidence. More long-term cohort studies reporting absolute risks and robust, well-conducted meta-analyses with dose-response information are needed before policy guidelines can be formed.

\section{Acknowledgements}

We would like to thank Dr Dena Zaraatkar for their advice regarding umbrella review methodology and use of the GRADE tool.

\section{References}

1. Dhanwal DK, Dennison EM, Harvey NC et al. (2011) Indian J Orthop 45, 15-22.

2. Veronese N \& Maggi S (2018) Injury 49, 1458-60.

3. Benetos IS, Babis GC, Zoubos AB et al. (2007) Injury 38, 735-44. 